\title{
El otro lado de la filosofía de la biología: una problematización de lo epistemológico como límite disciplinar
}

The other side of the philosophy of biology: a problematization of epistemology as a disciplinary limit.

Por: E. Joaquín Suárez-Ruíz ${ }^{*}$

Universidad Nacional de La Plata

CONICET

La Plata, Argentina

Email: ernestojoaquinsuarez@gmail.com

Fecha de recepción: 25/06/2018

Fecha de aprobación: 15/12/2018

Doi: http://dx.doi.org/10.30972/nvt.0153710

\section{Resumen}

El objetivo de este artículo es problematizar el supuesto de que la filosofía de la biología constituye una disciplina limitada al análisis epistemológico. Para ello se profundizará en las características de un tipo de análisis paralelo, llevado a cabo por cada vez más filósofos de la biología: aquel que toma los conocimientos biológicos como punto de partida para analizar problemas filosóficos, denominado aquí como "análisis epistémico". A lo largo de este desarrollo se contemplarán los paralelos gnoseológicos de estos dos tipos de análisis, y se los pondrá en relación con las críticas al supuesto filosófico de una excepcionalidad humana, realizadas por el filósofo francés Jean-Marie Schaeffer.

Palabras clave: filosofía de la biología, evolución biológica, tesis de la excepción humana, discontinuidad ontológica, filosofía continuista

\footnotetext{
* Magíster en filosofía por la Université Bordeaux-Montaigne (Francia). Licenciado en Filosofía, profesor en Comunicación Audiovisual y maestrando en Estética y Teoría de las Artes por la Universidad Nacional de La Plata (Argentina). Ha publicado sus investigaciones en revistas especializadas, ha participado de numerosos eventos científicos nacionales e internacionales, y forma parte de proyectos de investigación de la UNLP y de la Universidad de Buenos Aires (UBA). Actualmente es becario doctoral del Consejo Nacional de Investigaciones Científicas y Técnicas (CONICET, Argentina).
} 


\begin{abstract}
The objective of this article is to problematize the assumption that the philosophy of biology constitutes a discipline limited to the epistemological analysis. To this end, the characteristics of a type of parallel analysis, carried out by more and more philosophers of biology, will be deepened: that which takes biological knowledge as a starting point to analyze philosophical problems. Throughout this development, the gnoseological parallels of these two types of analysis will be contemplated, and they will be put in relation with the criticisms of the philosophical assumption of a human exceptionality, made by the french philosopher Jean-Marie Schaeffer.
\end{abstract}

Keywords: philosophy of biology, biological evolution, thesis of human exception, ontological discontinuity, continuist philosophy

\title{
Introducción
}

Tengo en mis estanterías cinco o seis volúmenes que incluyen en su título el rótulo engañoso de "filosofía de la ciencia". EI hecho real es que todos estos volúmenes son de filosofía de la física (Mayr, 1949).

La filosofía de la biología es una de las disciplinas filosóficas más recientes. Siendo que los tópicos de la filosofía de la ciencia general estaban muy influenciados por problemas ligados a la física, este campo del saber se consolida a principios de los años 70' ante la necesidad de dar lugar a una discusión que se centre en las características propias de las ciencias biológicas (Diéguez, 2012: 15). De este modo, términos como "adaptación", "gen" y "biodiversidad", adquirieron ciudadanía en el espacio de la discusión filosófica.

Entre la multiplicidad de conceptos, hay uno que es especialmente estudiado en las producciones ligadas a la filosofía de la biología, a saber, la noción de "evolución". A partir de la solidez teórica conseguida gracias a los desarrollos de Charles Darwin hacia fines del siglo XIX y a las numerosas investigaciones 
El otro lado de la filosofía de la biología:

una problematización de lo epistemológico como límite disciplinar

experimentales que la sustentan actualmente, la teoría de la evolución ${ }^{1}$ se estableció como una perspectiva que permite, tal como Dobzhansky lo resalta en su famosa frase, darle sentido a las ciencias biológicas como un todo. Justamente, su importancia reside allí: la teoría de la evolución, al menos en sus términos generales, se presenta como un supuesto necesario para la filosofía de la biología a la hora de analizar la mayoría de las nociones involucradas en la biología (Bunnin \& Yu, 2009: 522).

El ahondar en las características de los conocimientos biológicos en tanto problemas filosóficos renovó un vínculo que se había perdido. Justamente, si se examina la relación que los filósofos tuvieron con las ciencias biológicas desde los inicios de esta disciplina, se cae en la cuenta de que el imaginario del análisis filosófico como ajeno a este tipo de saberes experimentales, es propio de la filosofía contemporánea. Pensadores como Aristóteles, Descartes, Kant o Hegel desarrollaron sus filosofías a la par que estudiaban los descubrimientos científicos de su época.

Hacia fines del siglo $\mathrm{XX}$, junto a la renovación de este vínculo, muchos problemas que fueron tratados como propiedad exclusiva del campo filosófico comenzaron a mostrarse cada vez más comprensibles a la luz de la teoría de la evolución, que a través de la sola consulta de la historia de la filosofía. Así, en los últimos años surgieron investigaciones novedosas como las del neurocientífico Antonio Damasio respecto de los "marcadores somáticos" (Damasio, 1996), que señalan la posibilidad de comprender a las emociones como un tipo de cognición, o las del primatólogo Frans de Waal respecto de las "emociones morales", presentes también en primates no humanos (de Waal, 2007). Paralelamente, pensadores con formación filosófica como Daniel Dennet (1993), Philip Kitcher (2011) o Peter Godfrey-Smith (1996) empezaron a analizar problemas de la filosofía de la mente, de la filosofía del lenguaje o de la ética, tomando de base investigaciones fundadas en la teoría de la evolución.

\footnotetext{
${ }^{1}$ En filosofía de la biología se comprende que no hay algo así como "la" teoría de la evolución, sino que se trata más bien de un compendio de múltiples teorías sobre las que no hay consenso absoluto (Dupré, 2007: 28). Justamente, uno de los núcleos de discusión más activos en la actualidad es la perspectiva darwiniana de la evolución centrada en la selección natural. Véase, por ejemplo, Jablonka (2005), Margulis (2002) u Odling-Smee et al (2003). No obstante, a los fines de este artículo, continuaré utilizando el singular ("Ia" teoría) para referirme a una perspectiva evolutiva de lo humano en su relación con el resto de lo viviente.
} 
Teniendo en cuenta este contexto reciente, la filosofía de la biología muestra estar compuesta por dos tipos de análisis imbricados: uno epistemológico, cuyo acento se encuentra en la clarificación conceptual, y uno epistémico, el cual toma esos conocimientos como punto de partida para analizar problemas filosóficos. Las particularidades de esta doble constitución analítica de la disciplina en cuestión será el núcleo argumental a desarrollar en este artículo.

Ahora bien, a pesar del impacto actual de las investigaciones biológicas en el análisis de cuestiones pertenecientes a la filosofía, el análisis epistémico aún sigue siendo considerado marginal por gran parte de la comunidad filosófica. Aunque existen filósofos de la biología ligados a la tradición analítica ${ }^{2}$, como los mencionados más arriba, que resaltan la importancia filosófica de los saberes biológicos contemporáneos, lo más común de hallar en la literatura es el supuesto de que la filosofía de la biología debe limitarse al análisis epistemológico, dado que el introducir a las ciencias biológicas en el análisis de problemas filosóficos (no epistemológicos) implicaría, inevitablemente, caer en puntos de vista reduccionistas de lo humano. Según esta tendencia, perspectivas como las de Damasio o de Waal son comprendidas como miradas simplistas sobre fenómenos en donde la biología tan sólo puede realizar un aporte mínimo o ninguno en absoluto.

Sumados al puñado de teóricos vinculados a la filosofía analítica, actualmente hay varios pensadores pertenecientes a la tradición continental que hacen hincapié en la relevancia filosófica de la teoría de la evolución biológica ${ }^{3}$. Uno de los representantes más importantes de esta corriente es el intelectual francés Jean-Marie Schaeffer. Este filósofo afirma que detrás de la tendencia contemporánea a prescindir o, incluso, a denostar las investigaciones biológicas, subyace una herencia muy antigua de la historia de la filosofía: la idea de que hay algo ontológicamente excepcional en los seres humanos que permite comprenderlos -comprendernos- como una entidad

\footnotetext{
${ }^{2}$ La distinción entre la tradición analítica y la tradición continental de la filosofía se encuentra muy matizada actualmente. Es decir, no se trata de dos grupos rígidamente definidos, sino que existen numerosos cruces entre ambas. A pesar de ello, esta división continua siendo de utilidad para resaltar diferencias generales.

${ }^{3}$ Otro referente importante, en este caso de la tradición pragmatista de la filosofía, es John Dewey, quien ya a principios del siglo XX hacía hincapié en las profundas implicancias de la perspectiva evolutiva al interior de la filosofía (Dewey, 1997).
} 
El otro lado de la filosofía de la biología:

una problematización de lo epistemológico como límite disciplinar

radicalmente diferente del resto de los seres vivos. El filósofo denomina a esta concepción imbricada, no sólo en la filosofía, sino en el saber occidental en general, como "tesis de la excepción humana" (Schaeffer, 2009).

A partir de ese supuesto ontológico advertido por Schaeffer, se legitima, al mismo tiempo, un punto de vista historiográfico-disciplinar: el análisis filosófico debe centrarse en "lo propiamente humano", por lo que el estudio de las características compartidas con otros seres vivos son cuestiones accesorias o irrelevantes (Suárez, Anzoátegui \& Carrera, 2018). Partiendo de este supuesto, en las producciones filosóficas se da lo que Schaeffer denomina una "inmunización epistémica", es decir, la negación de que estudios provenientes de disciplinas centradas en el análisis de características naturales puedan echar luz sobre problemas filosóficos (Schaeffer, 2009: 25).

De manera que, si bien la metodología propia de la filosofía de la física podría comprenderse como un abordaje estrictamente epistemológico de las nociones utilizadas en la ciencia en cuestión, es decir, centrada en la clarificación conceptual, la metodología de análisis de la filosofía de la biología, siendo que la perspectiva evolutiva es uno de sus objetos de estudio prioritarios y que esta representa una lente imprescindible para fundamentar la continuidad ontológica entre los seres humanos y el resto de los seres vivos, plantea una problemática especial: la extensión epistémica de sus análisis permiten problematizar los supuestos discontinuistas que subyacen a las disciplinas filosóficas en general (Suárez, 2018).

Teniendo en cuenta este estado de la cuestión, en lo que sigue se argumentará sobre la relevancia del doble movimiento analítico inherente a la filosofía de la biología contemporánea, haciendo hincapié en el tipo de análisis paralelo al epistemológico. Es decir, en el epistémico: aquel que toma a los conocimientos biológicos como aportes relevantes en el estudio de problemas ligados a la ética, la estética, etc. Para ello, primero se analizarán los supuestos tras la tendencia a comprender la filosofía de la biología como reducida al análisis epistemológico, luego se ahondará en las características de la inmunización epistémica mencionada por Schaeffer y, finalmente, se examinarán algunas consecuencias a nivel disciplinar. 
La tesis a defender hacia el final del trabajo es que a las concepciones de la filosofía de la biología que centran su análisis exclusivamente en su costado epistemológico, es decir, a aquellas que niegan la relevancia de la biología contemporánea en el análisis de problemas filosóficos, legitiman, de manera directa o indirecta, la existencia de una discontinuidad ontológica entre lo humano y el resto de lo viviente.

\section{Lo epistémico y lo epistemológico}

El propósito de este parágrafo será desandar la idea de que la filosofía de la biología se limita al análisis epistemológico de los problemas de la biología, a través de una exploración de la distinción entre "lo epistemológico" y "lo epistémico". Aunque algunos diccionarios de filosofía los tratan como conceptos equivalentes (Blackburn, 1996: 154), es posible hallar tras ella un paralelo gnoseológico implícito que permitirá fundamentar la importancia del doble movimiento analítico de la filosofía de la biología. Para ello se analizará la base etimológica y gnoseológica tras dicha separación.

Respecto de "lo epistemológico", sin profundizar demasiado en los aspectos polisémicos, se comprende que la unión entre los términos griegos episteme y logos refiere a la explicación, reflexión o discurso sobre el conocimiento (Audi, 1999: 273). Por su parte, "lo epistémico", al tener como raíz sólo el término episteme, refiere al nivel del conocimiento en sí mismo (Lacey, 1996: 96). Este último generalmente se utiliza como epíteto de otra noción. Por ejemplo, el concepto "justificación epistémica" refiere a la evidencia ${ }^{4}$ que fundamenta un conocimiento determinado (Audi, 1999: 275).

Yendo ahora al trasfondo gnoseológico, resulta preciso realizar una breve genealogía histórica. Las posiciones más extremas respecto de la naturaleza del conocimiento provienen de la antigua división entre el dogmático y el escéptico. Se trata de dos posturas abstractas, inexistentes en lo concreto, pero muy explicativas en

\footnotetext{
${ }^{4}$ Diferente del concepto de "verdad", esta admite gradación en la posibilidad del conocimiento: Epistemic justification, unlike truth, is capable of degrees of the things that we are justified in believing; some are more justified than others (Bunnin \& Yu, 2009: 217). Destaco esto por el hecho de que se supondrá dicha noción en lo que sigue.
} 
El otro lado de la filosofía de la biología:

una problematización de lo epistemológico como límite disciplinar

lo que refiere a los extremos en el orden gnoseológico. Por un lado, el dogmático es aquel que afirma que es posible conocer la verdad en términos absolutos. Por otro lado, el escéptico no se compromete con ninguna afirmación de tipo dogmática, sino que suspende el juicio ante la cuestión de si existe o no la verdad y tan sólo utiliza argumentos para llevar hacia la aporía al escéptico (Popkin, 1983). En palabras de la filósofa Cristina Di Gregori:

El dogmático es aquel que sostiene que podemos determinar la verdadera naturaleza de las cosas en base a nuestras capacidades cognoscitivas. Vale decir, podemos tener genuino conocimiento del mundo en términos de creencias verdaderas y justificadas. El escéptico ${ }^{5}$ considera que el dogmático afirma la real existencia de aquello acerca de lo cual dogmatiza -en el sentido de aprobar un asunto- mientras que él mismo no propone ninguna de sus expresiones como si lo manifestado en ellas existiera de modo absoluto. El escéptico al formular sus tropos o aporías muestra la inviabilidad de un conocimiento genuino del mundo. La solidez cognoscitiva que pretende el dogmático sería irrealizable. Vale decir, la imposibilidad de preferir entre opiniones contrarias sobre una misma cuestión. Esto los conduce a proponer la suspensión del juicio. (Di Gregori, 1996: 411)

Si bien representan posturas opuestas, el escéptico y el dogmático se necesitan uno al otro en su vinculación con el conocimiento. El dogmático precisa del escéptico a la hora de argumentar la existencia de una verdad absoluta, dado que sin él no cabría ninguna duda en su certeza. El escéptico, por su parte, precisa del dogmático por el hecho de que su argumentación depende de aquel que dice estar parado sobre una base sólida. En este desarrollo, el interés en estas posiciones gnoseológicas extremas e interdependientes reside en que, a partir de ellas, será posible comprender mejor la complementariedad entre lo epistemológico y lo epistémico.

\footnotetext{
${ }^{5}$ Siguiendo la interpretación de Di Gregori, se comprende que el rol del escéptico propiamente dicho sería el del escéptico pirrónico. El escéptico académico, al afirmar que no es posible conocer la verdad, se compromete con un dogmatismo negativo.
} 
Justamente, al mantener este nivel gnoseológico abstracto es posible constatar que, por un lado, "lo epistémico" -tomado no como epíteto sino como sustantivo, es decir, como aquello referido al conocimiento en sí mismo- sería más propio del abordaje cognoscitivo del dogmático. Esto es, el dogmático supone que es posible hablar del conocimiento en sí mismo y comprenderlo como un fundamento firme. Por otro lado, "lo epistemológico" sería un tipo de análisis ligado a la postura del escéptico, el cual no se compromete con ningún tipo de pretensión de verdad en el conocimiento, sino que coteja las múltiples aristas de la cuestión de manera distanciada.

Ahora bien, cabe hacer una salvedad importante: aunque el análisis epistemológico y el rol del escéptico sean posturas estrechamente ligadas, no son idénticos. El análisis de tipo epistemológico también puede comprenderse como fundado en presupuestos dogmáticos. Un ejemplo de ello, oportuno para nuestra investigación, es lo ocurrido entre el concepto de antropomorfismo y el de antroponegación. El primero remite a una precaución metodológica en biología del comportamiento respecto de, a la hora de describir sus conductas, no investir a los animales no humanos con características humanas. La segunda noción fue propuesta por Frans de Waal para advertir que, paradójicamente, dicha precaución metodológica corre el riesgo de legitimar el supuesto ontológico de una discontinuidad absoluta de las características humanas. En palabras de de Waal:

Necesitando un nuevo término para expresar mi punto de vista, he inventado la "antroponegación", que es el rechazo a priori de rasgos similares a los humanos en otros animales o rasgos similares a nosotros. El antropomorfismo y la antroponegación tienen una relación inversa: cuanto más cerca esté de nosotros otra especie, más antropomorfismo ayudará a nuestra comprensión de esta especie y mayor será el peligro de antroponegación. Por el contrario, cuanto más distante esté una especie de nosotros, mayor será el riesgo de que el antropomorfismo proponga similitudes cuestionables que han surgido de forma independiente ${ }^{6}$. (de Waal, 2017: 52)

\footnotetext{
${ }^{6}$ La traducción es mía, al igual que la de la siguiente cita de de Waal.
} 
Así, si bien afirmar que las hormigas tienen "reinas", "soldados" y "esclavos" es una caracterización antropomórfica, negar cualidades compartidas con animales mucho más cercanos en términos evolutivos, como por ejemplo los chimpancés, implica una antroponegación.

En los términos que vienen siendo planteados, aunque el antropomorfismo sería un recaudo epistemológico importante para no caer en afirmaciones absurdas como "la ardilla está reflexionando", cuando se convierte en la justificación de una discontinuidad radical, revela poseer en sí la posibilidad de convertirse en una concepción dogmática. En este caso, el análisis de tipo epistémico se vuelve un mediador indispensable para explicitar los posibles abusos de un análisis que se pretende exclusivamente epistemológico. Por ejemplo, a través de observaciones de cómo los chimpancés al hacerles cosquillas hacen "sonidos entrecortados con un ritmo de inhalación y exhalación que se asemeja a la risa humana" (de Waal, 2017: 52), y teniendo en cuenta -nuestra- cercanía evolutiva para con ellos, de Waal puede argumentar sobre la necesidad de una advertencia metodológica paralela a la tradicional en biología del comportamiento. Justamente, es gracias a este tipo de investigaciones experimentales (epistémicas) que el primatólogo permite dar cuenta de un recaudo epistemológico complementario al "antropomorfismo", a saber, la "antroponegación".

Aunque en este punto del desarrollo aún resulte una mención demasiado abstracta, puede decirse que este trasfondo gnoseológico resulta fundamental para comprender por qué cuando se considera la filosofía de la biología como una empresa exclusivamente epistemológica se corre el riesgo de legitimar una concepción discontinuista de los seres humanos. Hasta aquí es posible afirmar que, cuando la filosofía de la biología supone que puede mantenerse exclusivamente en el rol del escéptico a partir de considerar que su límite disciplinar se encuentra en lo epistemológico, reproduce de manera subrepticia dogmas no reconocidos como tales, de manera análoga a lo que ocurría en el ejemplo de la complementariedad metodológica entre "antropomorfismo" y "antroponegación”. Para ahondar en ello, en 
el próximo parágrafo se expondrán algunos problemas que se desprenden de considerar la filosofía de la biología como limitada al nivel epistemológico.

\section{“Filosofía de...": cuando la filosofía se considera un espacio neutro}

Tal como fue mencionado en la introducción, la concepción de la filosofía de la biología en tanto una empresa exclusivamente epistemológica pierde de vista una cuestión importante: la teoría de la evolución biológica es la perspectiva más sólida para fundamentar la existencia de una continuidad ontológica entre los humanos y el resto de los seres vivos. La comprensión del porqué de este desinterés o, incluso, del rechazo a priori por parte de no pocos filósofos de la biología al análisis epistémico, parece poseer varias causas.

En parte se vincula al distanciamiento analítico que le es propio a la filosofía de la ciencia contemporánea desde principios del siglo XX (Audi, 199: 956). A esto se le sumó, hacia la segunda mitad del mismo siglo, un distanciamiento de tipo sociológico y antropológico. A partir de obras famosas como las de Thomas Kuhn (1962), Paul Feyerabend (1974) o Bruno Latour (1979), especialmente por sus críticas a los supuestos de neutralidad e imparcialidad en las ciencias, gran parte de los filósofos de la ciencia centraron su atención en los diferentes intereses que subyacen a las instituciones científicas: políticos, económicos e ideológicos.

Más allá de la novedad que representaron en su época, y siguiendo la caracterización amplia realizada en el apartado anterior, es posible incluir estos análisis antropológicos y sociológicos de la ciencia en la categoría de "lo epistemológico", por el hecho de que en la aproximación a su objeto de estudio buscan evitar todo compromiso con los saberes que analizan. Es decir, en términos gnoseológicos, pretenden poseer una cercanía mayor al rol del escéptico que al del dogmático.

Respecto de la filosofía de la biología, si bien habría consolidado su independencia disciplinar a principios de los años 70', continuaría con la tendencia metodológica característica de la filosofía de la ciencia centrada en la física: dedicarse exclusivamente al análisis epistemológico. Tal como se mencionó en la introducción, 
El otro lado de la filosofía de la biología:

una problematización de lo epistemológico como límite disciplinar

aunque haya filósofos de la biología que realizan análisis de tipo epistémico, el status quo ha venido siendo el anclaje exclusivo en lo epistemológico.

Lo que subyace a esta concepción instalada es una dificultad ya advertida más arriba: suponen que el análisis epistemológico y el rol del escéptico coinciden enteramente, y que pueden desentenderse del aspecto dogmático. Es decir, el problema surge cuando se considera que la filosofía de la biología, en tanto disciplina cuyo análisis tiende más a lo epistemológico que a lo epistémico $y$, a nivel gnoseológico, más al rol del escéptico que al del dogmático, puede desempeñar de manera exclusiva el rol del escéptico. Cuando esto ocurre, tal como se adelantó con el ejemplo del "antropomorfismo", los análisis epistemológicos que se suponen ajenos a influencias dogmáticas corren el riesgo, justamente, de legitimar y reproducir dogmas de manera subrepticia.

Esto se representa bien en los enfoques de la filosofía de la biología que centran sus análisis exclusivamente en una aproximación de tipo antropológica o sociológica: aunque múltiples investigaciones vinculadas a la neurociencia, a la psicología experimental o a la primatología estén modificando el concepto de lo humano tanto a nivel individual como social, numerosos de sus representantes todavía mantienen enfoques fundados en supuestos antropológicos y sociológicos de principios del siglo pasado, presumiendo que los estudios recientes en nada afectan a su punto de vista ${ }^{7}$. De modo que la inclusión de las perspectivas antropológicas y sociológicas de la ciencia entre los filósofos de la biología parece no sólo no haber puesto en duda el status quo de "lo epistemológico", sino que, aún más, parece haber tendido a fortalecerlo.

Paradójicamente, si bien los defensores de este tipo de enfoques antropológicos y sociológicos de la filosofía de la biología confiarían en estar haciéndole frente a las posiciones epistemológicas positivistas que suponen al campo científico como inalterado por las influencias sociales, estarían fortaleciendo, por otro lado, la convicción de que la filosofía de la biología sí tendría permitido comprenderse

\footnotetext{
${ }^{7}$ Aquí se explicita aún más el porqué es posible agrupar a los enfoques antropológicos y sociológicos de la ciencia en la categoría amplia de "lo epistemológico". Justamente, porque suponen que su abordaje distanciado de los saberes biológicos se asienta sobre una base en absoluto comprometida con dichos conocimientos.
} 
como un espacio neutro e imparcial. Muy por el contrario, al suponer que es posible asestar críticas a las ciencias biológicas sin comprometerse con ninguna pretensión de verdad, los enfoques de tipo antropológico y sociológico de la filosofía de la biología se instalan firmemente en un punto de vista fundado en, siguiendo a Jean-Marie Schaeffer, la tesis de la excepción humana. Más allá de que resulte más evidente en estos enfoques, el problema en cuestión podría extenderse a toda concepción de la filosofía de la biología que asevere no poseer supuestos dogmáticos.

Habiendo llegado a este punto, es posible señalar el porqué lo epistemológico como límite disciplinar representa un inconveniente para la filosofía de la biología en particular, y para la filosofía en general. Tal como se desarrolló en el apartado anterior, al contemplar su doble movimiento analítico, la filosofía de la biología muestra poseer aspectos gnoseológicos ligados tanto al rol del dogmático como al del escéptico. A su vez, se advirtió que un análisis de tipo epistemológico también puede comprenderse como fundado en presupuestos dogmáticos. En relación a esto, cuando se tiene en cuenta la situación señalada por Schaeffer, se distingue que la tesis de la excepción humana perjudicaría a la filosofía en sentido amplio, incluida la filosofía de la biología.

En consecuencia, siendo que la filosofía de la biología es la disciplina filosófica que se ocupa de la teoría de la evolución, y que esta teoría representa la perspectiva más sólida para justificar la continuidad ontológica entre los seres humanos y el resto de los seres vivos, descuidar el análisis de tipo epistémico implica descuidar la posibilidad de argumentar seriamente sobre la continuidad ontológica. Entonces, a la supuesta exclusividad de lo epistemológico y prescindencia de lo epistémico en la filosofía de la biología subyace un dogma firmemente arraigado: la discontinuidad ontológica de lo humano respecto del resto de los seres vivos.

Así, la negación del análisis epistémico conduce a la desestimación a priori de los posibles aportes que los conocimientos biológicos podrían ofrecer a otras subdisciplinas filosóficas para subsanar la brecha ontológica mencionada y, como consecuencia, implica el enraizamiento de supuestos dogmáticos (de distintos ordenes: antropológicos, sociológicos, etc.). Tal como se anticipó en la introducción, esta tendencia se encuentra vinculada a lo que Schaeffer denomina inmunización 
El otro lado de la filosofía de la biología:

una problematización de lo epistemológico como límite disciplinar

epistémica: al suponer la tesis de la excepcionalidad, el enfoque puramente epistemológico de la filosofía de la biología se considera inmunizado frente a conocimientos que provienen de las ciencias biológicas, suponiendo que estas no afectan en nada la lente con la que analiza la realidad (Schaeffer, 2009: 25).

De modo que, si en la filosofía de la biología no se tiene en cuenta que el rol del dogmático representa una figura inherente a su constitución, la denominación "filosofía de..." se convierte en una trampa discursiva. Legitima la idea de que, gracias a su amparo epistemológico, esta disciplina puede tratarse a sí misma como un ámbito neutro e imparcial. Lo que genera este status quo epistemológico es, en realidad, la reproducción del supuesto pre-darwiniano de una discontinuidad absoluta de lo humano o, en términos de Schaeffer, de la tesis de la excepción humana.

\section{Hacia una concepción continuista de la filosofía}

Lo central en esta última y breve sección será hacer hincapié en una cuestión que se desprende del desarrollo anterior, pero cuya explicitación no se encuentra comúnmente entre las producciones ligadas a la filosofía de la biología. Nuevamente, si bien es usual hallar en los textos de filósofos de la biología como Dennett, Kitcher o Godfrey-Smith una complementariedad entre el análisis epistemológico y el epistémico, en sus desarrollos no suele encontrarse el énfasis en un hecho muy simple pero sumamente importante a nivel disciplinar: cuando la filosofía de la biología abarca el nivel epistémico de análisis, renovando así el vínculo entre filosofía y biología que sí poseían filósofos como Aristóteles, Descartes o Kant, y da lugar a nuevas respuestas sobre el origen de la moral, la experiencia estética o el lenguaje, su análisis se extiende más allá de esta sub-disciplina.

¿Por qué lo propiamente filosófico no debe incluir conocimientos biológicos?, ¿hasta qué punto la filosofía puede considerar como relevantes argumentos de la historia de la filosofía que suponen una discontinuidad ontológica de lo humano?, ¿qué justifica que la filosofía continúe circunscribiendo sus análisis a "lo propiamente humano" sino el supuesto de que hay algo ontológicamente "excepcional" en este ser? Son algunas de las preguntas que surgen de esta demanda, llevada a cabo por parte 
por cada vez más filósofos de la biología, respecto de la necesidad de considerar la relevancia de las ciencias biológicas en el análisis filosófico.

Así, una vez que la investigación en filosofía de la biología se extiende hacia problemas que tradicionalmente pertenecieron a otras sub-disciplinas como la ética, la estética, la filosofía de la mente o la filosofía del lenguaje, lo que señala son problemas de tipo meta-filosófico. Es decir, los problemas que abarca ya no son solamente "filosofía de la biología" sino, ante todo, "meta-filosofía": la interrogación sobre la constitución de la filosofía misma. Una vez que da este salto, la filosofía de la biología pasa a señalar un problema que abarca a las disciplinas filosóficas en general, a saber, la necesidad de pensar en profundidad cómo sería posible una filosofía continuista. Esto es, una concepción de la filosofía que tenga en cuenta a la teoría de la evolución como un cimiento indispensable a la hora de justificar una continuidad ontológica entre los seres humanos y el resto de los seres vivos.

Sin dudas, el considerar a la perspectiva evolutiva como un cimiento indispensable a la hora de hacer filosofía en la actualidad conlleva numerosos problemas complementarios que precisarían un extenso desarrollo aparte. Aquí me limitaré a mencionar un punto que se desprende de lo ya expuesto, pero que resaltaré brevemente: en la búsqueda de una concepción continuista de la filosofía, el hecho de que resulte indispensable suponer la teoría de la evolución para fundamentar la continuidad ontológica, no implica que esta búsqueda pueda desentenderse de su análisis epistemológico complementario. Es decir, en términos gnoseológicos, se trata de un supuesto dogmático que no se desentiende del rol del escéptico. Es en esto en lo que consiste, justamente, el feedback analítico entre los análisis epistémico y epistemológico que posibilita la filosofía de la biología. En definitiva, a pesar de las complicaciones derivadas, una vez develada la reproducción constante de supuestos discontinuistas en la filosofía, dichos problemas no son pretexto para no dar lugar al debate sobre la posibilidad de una filosofía continuista ${ }^{8}$.

\footnotetext{
8 Una breve aclaración sobre las implicancias meta-filosóficas: dada la relevancia del análisis epistemológico a la hora de suponer conocimientos biológicos en la investigación de problemas filosóficos, es que resulta preferible considerar a la "filosofía continuista" como una perspectiva metafilosófica derivada del doble movimiento analítico inherente a la filosofía de la biología. Proyectos meta-
} 
El otro lado de la filosofía de la biología:

una problematización de lo epistemológico como límite disciplinar

\section{Conclusiones}

Tal como sugiere Mayr en el epígrafe que inicia este artículo, la filosofía de la biología surgió como reacción ante el hecho de que la mayor parte de la literatura sobre filosofía de la ciencia era, más bien, filosofía de la física. Esta equiparación implícita impedía que las características de las ciencias biológicas pudiesen ser analizadas con propiedad, es decir, resultaba preciso librar a esta disciplina del supuesto de que las características epistemológicas de la física podrían reducir las características epistemológicas de otras ciencias como, en este caso, la biología. Dada la gran cantidad de producciones y grupos de investigación que actualmente se dedican a la filosofía de la biología, la herencia de la filosofía de la física tras el engañoso título de "filosofía de la ciencia" ya no representa un problema para el nivel epistemológico de esta disciplina, pero, teniendo en cuenta su escasez, sí parece continuar siéndolo para su extensión epistémica. Así, según lo que ha sido desarrollado a lo largo de este artículo, se evidencia que aún subyace a gran parte de la literatura dedicada a la filosofía de la biología una idea heredada de la filosofía de la física: que su propósito es (y debe ser) el de limitarse a la clarificación conceptual y al análisis distanciado que caracteriza a la epistemología tradicional.

El objetivo de este trabajo fue el de realizar una aproximación a dos cuestiones marginales en las producciones ligadas a la filosofía de la biología. Por un lado, señalar la relevancia de pensar la filosofía de la biología como constituida por un análisis doble que se complementa constantemente. Por otro, a partir de considerar las extensiones del análisis epistémico, remarcar la relevancia de pensar una filosofía continuista. Es decir, resulta fundamental el fomentar la proliferación de investigaciones vinculadas a este tipo de análisis, para así advertir la potencialidad de la filosofía de la biología a la hora de problematizar la reproducción de la tesis de la excepción humana al interior de la filosofía.

Finalmente, a partir de esta necesidad de pensar una filosofía continuista, se acentúa aún más la relevancia de comprender a la filosofía de la biología como

filosóficos como la "filosofía científica" de Mario Bunge y discípulos, suelen incurrir en el descuido del análisis epistemológico de la ciencia, sobre todo del nivel antropológico y sociológico. 
compuesta por un doble análisis epistemológico-epistémico, ya que se revela como el espacio propicio para dar lugar a una complementariedad crítica entre biología y filosofía: las ciencias biológicas, por un lado, precisan del análisis filosófico para problematizar las influencias sociales, históricas y económicas en sus instituciones; por otro lado, la filosofía precisa de las ciencias biológicas para problematizar los supuestos discontinuistas heredados de la historia de la filosofía, que aún suelen ser reproducidos como saberes vigentes ${ }^{9}$.

\section{Bibliografía}

Audi, Robert. The Cambridge Dictionary of Philosophy. Cambridge, Cambridge University Press, 1999.

Bartley, W. W. "Philosophy of biology versus philosophy of physics". En G. Radnitzky y W. W. Bartley III (eds.), Evolutionary epistemology, rationality, and the sociology of knowledge, La Salle, Illinois: Open Court: 7-14, 1987.

Blackburn, Simon. The Oxford Dictionary of Philosophy. Oxford, Oxford University Press, 1996.

Bunnin, Nicholas, Yu, Jiyuan. The Blackwell Dictionary of Western Philosophy. New Jersey, Wiley-Blackwell, 2009.

Caponi, Gustavo. “¿Qué quiere decir "naturalizar”?”. En Ludus Vitalis, vol. XXVI, núm. 50, 2018.

Damasio, A. El Error de Descartes. Santiago de Chile, Andrés Bello, 1996.

Dennett, Daniel. La Peligrosa Idea de Darwin. Barcelona, Galaxia Gutenberg, 1999.

Dewey, John. The influence of Darwin on Philosophy and other essays. Nueva York, Prometheus Books, 1997.

Di Gregori, María Cristina, "Reflexiones sobre escepticismo y relativismo", en Actas de las 1o Jornadas de Investigación para Profesores, Graduados y Alumnos (FaHCE, UNLP), 1996.

\footnotetext{
${ }^{9}$ No se trata de "naturalizar" la filosofía (Caponi, 2018), sino, más bien, de que la filosofía de la biología y los filósofos en general nos hagamos cargo por fin de las implicancias que posee en el análisis filosófico una concepción continuista de los seres humanos. Es decir, en pocas palabras, de hacer filosofía en el siglo XXI.
} 
El otro lado de la filosofía de la biología:

una problematización de lo epistemológico como límite disciplinar

Diéguez, Antonio. La vida bajo escrutinio. Una introducción a la filosofía de la biología. Barcelona, Biblioteca Buridán, 2012.

Godfrey-Smith, Peter. Complexity and the Function of Mind in Nature. Nueva York, Cambridge University Press, 1996.

Jablonka, E. \& Lamb, M. J. Evolution in four dimensions: genetic, epigenetic, behavioral and symbolic variation in life history. Cambridge, MIT Press, 2005.

Kitcher, Philip. The Ethical Project. Londres, Harvard University Press, 2011.

Lacey, Alan. A Dictionary Of Philosophy. Londres, Routledge, 1996.

Margulis, Lynn. Planeta Simbiótico. Un nuevo punto de vista sobre la evolución. Madrid, Editorial Debate, 2002.

Odling-Smee, F. J., Laland, K. N. \& Feldman, M. W. Niche construction: the neglected process in evolution. Princeton University Press, 2003.

Popkin, Richard. La historia del escepticismo desde Erasmo hasta Spinoza. Buenos Aires, Fondo de Cultura Económica, 1983.

Schaeffer, Jean Marie. El fin de la excepción humana. Buenos Aires, FCE, 2009.

Suárez, Ernesto Joaquín. "Para una caracterización filosófica de la continuidad evolutiva". En Revista Latinoamericana de Estudios Críticos Animales, Año V, Volumen II, 2018.

Suárez, Ernesto Joaquín; Carrera, Luciana y Anzoátegui, Micaela. “Adiós a la torre de marfil o sobre cómo abandonar la tesis de la excepción humana en la filosofía". En Revista Latinoamericana de Estudios Críticos Animales, Año V, Volumen I, 2018.

Waal, Frans de. Primates y filósofos: la evolución de la moral del simio al hombre. Barcelona, Paidós, 2007.

---Are we smart enough to know how smart animals are. Nueva Yrok, Norton \& Company, 2016. 